and removes the distinct DNA sequences found between the $\mathrm{V}, \mathrm{D}$ and J gene segments, tightly coordinating the process to ensure that different types of segment are subsequently connected. In cells, RAG also largely stops the removed DNA from being reinserted elsewhere in the genome, to prevent potentially harmful changes to the genetic code. But how did these functions evolve? A logical proposal implicates RAG2, but a recent study ${ }^{11}$ of ProtoRAG - a relative of RAG found in invertebrates that contains RAG 2 but still acts as a transposase - shows that things are more complicated. Elements in both RAG1 and RAG2 help to coordinate DNA cleavage and prevent insertion.

Liu and colleagues' findings cast fresh light on the role of RAG2, showing that it carries out many of the functions of $\mathrm{ZnB}$, but increases the rigidity of the whole RAG complex, compared with that of the transposase complex. It binds the DNA at the $V$, D and J segments more tightly than $\mathrm{ZnB}$ binds at the transposon, and does not undergo such large conformational changes (which can require a lot of energy, and thus reduce efficiency). This increased rigidity and tight binding might help to ensure the strict molecular coordination required for $\mathrm{V}(\mathrm{D}) \mathrm{J}$ recombination. It might also prevent release of cleaved DNA segments and/or stop the wings from reopening to accept any other DNA molecules - thereby preventing removed DNA from being reinserted elsewhere in the genome. If the wings do not open, then any incoming DNA would have to bend itself to an angle of about $150^{\circ}$ before entering the protein, which is not easily done.

Note that Liu et al. were not able to directly observe the structure of the transposase in complex with intact target DNA. It therefore remains to be seen whether target DNA first binds to the transposase in a relaxed form and is then forced into a severe $150^{\circ}$ bend. The authors also did not observe a complex in which the transposase binds intact transposon DNA such that the catalytic core is close enough to the ends to cleave them; instead, the authors observed intact transposon DNA bound with its ends away from the catalytic centre. In RAG, a large twist in the DNA is needed to position its breakpoints accurately for the cuts ${ }^{14}$. A similar twist might occur in Transib, but other explanations are also possible.

Efforts are now needed to define the exact functions of RAG2. Curiously, the cell-free RAG complex can readily insert excised DNA into another DNA molecule ${ }^{17,18}$ (a target DNA). Structures of RAG with a bound target DNA must therefore be obtained - ideally, both with the intact target and after insertion. These structures will show whether the target DNA becomes as sharply bent as it does in the Transib transposase, and reveal how RAG 2 affects the binding of target
DNA and its insertion of excised DNA.

Other proteins might be needed to promote the function of RAG. This possibility has previously been investigated, but the availability of new structures and methods provides further opportunities for research. For example, large molecular assemblies can now be studied inside cells using a technique called electron tomography ${ }^{19}$, and molecular interactions can be probed with advanced massspectrometry methods ${ }^{20}$. Analysis of genomic data from different species will also be helpful in identifying ancestors of RAG proteins other than ProtoRAG and the Transib transposase, and thereby exploring their evolutionary history. Such research will help to explain how parasitic genetic elements can be repurposed for crucial biological functions.
Orsolya Barabas is in the Structural
and Computational Biology Unit,
European Molecular Biology Laboratory, 69117 Heidelberg, Germany.
e-mail: barabas@embl.de

1. Lander, E. S. et al. Nature 409, 860-921 (2001).

2. Bourque, G. et al. Genome Biol. 19, 199 (2018)

3. Jones, J. M. \& Gellert, M. Immunol Rev. 200, 233-248 (2004).

4. Liu, C., Yang, Y. \& Schatz, D. G. Nature 575, 540-544 (2019)

5. Kapitonov, V. V. \& Jurka, J. PLoS Biol. 3, e181 (2005).

6. Hickman, A. B. \& Dyda, F. Microbiol. Spectr. 3 MDNA3-0034-2014 (2015).

7. Davies, D. R., Goryshin, I. Y., Reznikoff, W. S. \& Rayment, I. Science 289, 77-85 (2000)

8. Richardson, J. M., Colloms, S. D., Finnegan, D. J. \& Walkinshaw, M. D. Cell 138, 1096-1108 (2009).

9. Hickman, A. B. et al. Nucleic Acids Res. 46, 10286-10301 (2018).

10. Montaño, S. P., Pigli, Y. Z. \& Rice, P. A. Nature 491, 413-417 (2012).

11. Zhang, Y. et al. Nature 569, 79-84 (2019).

12. Ru, H. et al. Cell 163, 1138-1152 (2015).

13. Kim, M.-S. et al. Mol. Cell 70, 358-370 (2018).

14. Ru, H. et al. Nature Struct. Mol. Biol. 25, 732-742 (2018).

15. Kim, M.-S., Lapkouski, M., Yang, W. \& Gellert, M. Nature 518, 507-511 (2015).

16. Chen, S. \& Li, X. Gene 408, 51-63 (2008)

17. Hiom, K., Melek, M. \& Gellert, M. Cell 94, 463-470 (1998).

18. Agrawal, A., Eastman, Q. M. \& Schatz, D. G. Nature 394, 744-751 (1998).

19. Mahamid, J. et al. Science 351, 969-972 (2016).

20. Smits, A. H. \& Vermeulen, M. Trends Biotechnol. 34 825-834 (2016).

\title{
Astrophysics
}

\section{Extreme emission seen from $\gamma$-ray bursts}

Bing Zhang

Cosmic explosions called $\gamma$-ray bursts are the most energetic bursting events in the Universe. Observations of extremely high-energy emission from two $\gamma$-ray bursts provide a new way to study these gigantic explosions. See p.455, p.459 \& p.464

Astrophysical explosions known as $\gamma$-ray bursts (GRBs) can release in one second the amount of energy that the Sun will produce in its entire lifetime ${ }^{1}$. The emission from GRBs covers a broad stretch of the electromagnetic spectrum and occurs in two stages: the prompt-emission phase and the afterglow phase. The main emission mechanism is thought to be synchrotron radiation, whereby the gyration of energetic electrons around magnetic-field lines releases photons. Until now, emission from GRBs has been observed only at energies below 100 gigaelectronvolts $(\mathrm{GeV})$. Three papers in this issue $\mathrm{e}^{2-4}$ report observations of $\gamma$-rays that have energies above $100 \mathrm{GeV}$ from two bright GRBs, dubbed GRB 190114C and GRB 180720B.

The Major Atmospheric Gamma Imaging Cherenkov (MAGIC) Collaboration ${ }^{2}$ (page 455) detected photons in the teraelectronvolt range $\left(1 \mathrm{TeV}\right.$ is $10^{3} \mathrm{GeV}$ ) from GRB $190114 \mathrm{C}$, using the MAGIC telescopes at La Palma, Spain. The first detections started about one minute after the burst triggered NASA's two spaceborne GRB detectors: the Burst Alert Telescope on board the Swift satellite and the Gamma-ray Burst Monitor on board the Fermi satellite. The high-energy photons continued to rain down on the MAGIC telescopes for about 20 minutes, with the flux decreasing rapidly over this time. The MAGIC Collaboration and colleagues $^{3}$ (page 459) detected this GRB using several other ground-based and space-borne telescopes. When combined with the MAGIC data, this rich data set allowed the authors to model the event comprehensively and study how the TeV emission was produced.

Abdalla et al. ${ }^{4}$ (page 464) detected photons of energies above $100 \mathrm{GeV}$ (but below $1 \mathrm{TeV}$ ) from GRB 180720B, using the High Energy Stereoscopic System (HESS) array of telescopes in Namibia. Although these photons were lower in energy and fewer in number than those observed from GRB 190114C, they were detected from deep in the afterglow phase (10 hours after the GRB was triggered and 
lasting for 2 hours). The flux and maximum energy of the afterglow emission both decrease over time, owing to deceleration of the jets - the two narrow, oppositely directed channels through which most of the explosive energy of a GRB is released. Consequently, the detection of such high-energy photons deep in the afterglow phase is also groundbreaking.

The MAGIC and HESS observatories both use an array of optical telescopes called imaging atmospheric Cherenkov telescopes (IACTs), which are designed to detect $\gamma$-rays in the very-high-energy range (roughly from $30 \mathrm{GeV}$ to $100 \mathrm{TeV}$ ). More precisely, the IACTs detect the light (known as Cherenkov radiation) that is produced when such $\gamma$-rays hit Earth's atmosphere and produce a shower of charged particles. These facilities have been operating for more than a decade. GRBs, as the most powerful explosions in the Universe, have been one of the main observational targets, but, until now, have evaded detection. The current results are therefore a triumph for these observatories.

The discoveries are also a triumph for GRB theories. Theoretically, there are three mechanisms by which high-energy $\gamma$-rays can be produced during the afterglow phase ${ }^{5}$. The first is synchrotron radiation from electrons accelerated by the external shock - the shock wave that is generated when the exploded matter collides with surrounding interstellar gas. This emission component has a maximum energy that depends only on the Lorentz factor of the outflow (a parameter that denotes how fast the external shock is moving). To reach energies above $100 \mathrm{GeV}$, the Lorentz factor must be greater ${ }^{5}$ than about 1,000 , which is only marginally possible. Observations show that the Lorentz factor of GRB jets is usually a few hundred during the prompt-emission phase and decreases over time during the afterglow phase ${ }^{6}$.

The second high-energy radiation mechanism is synchrotron radiation from protons accelerated by the GRB external shock. This emission component can, in principle, contain $\mathrm{TeV} \gamma$-rays. However, because protons are much less efficient emitters than are electrons, the conditions for this mechanism to be dominant are rather demanding. Finally, the third mechanism is called synchrotron self-Comp$\operatorname{ton}^{7}$ (SSC), whereby the same accelerated electrons that emit synchrotron photons can scatter off some of these photons, resulting in photons that have energies above $100 \mathrm{GeV}$ (Fig. 1a). For typical shock-microphysics parameters inferred from afterglow modelling of other GRBs, it is expected that the SSC mechanism should usually be the main way in which high-energy $Y$-rays are produced ${ }^{5}$.

One key prediction of the SSC mechanism is that there should be two 'humps' in the spectral energy distribution of the afterglow spectrum ${ }^{5,7}$ (Fig. 1b). Such a two-hump

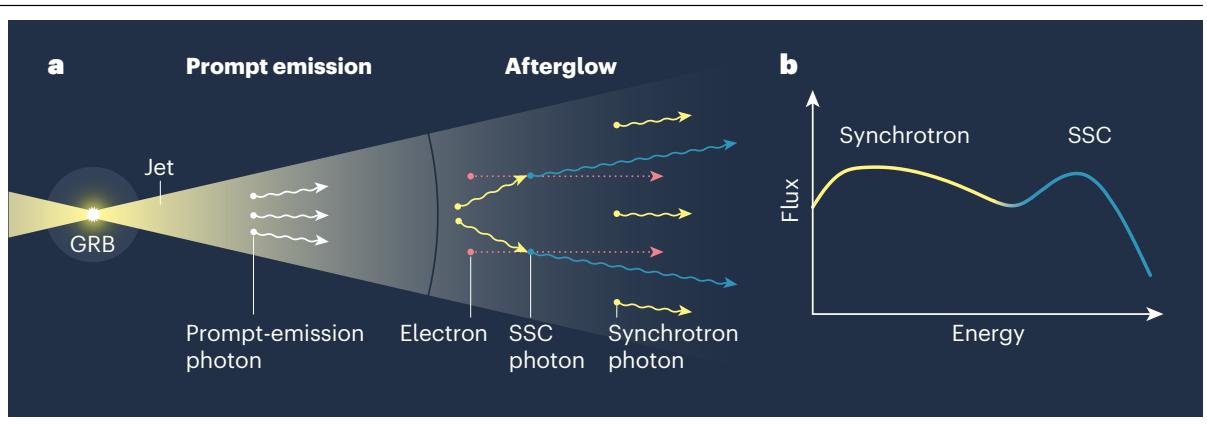

Figure 1 | Emission from a $\mathbf{y}$-ray burst. a, Three papers ${ }^{2-4}$ report the detection of high-energy radiation from astrophysical explosions known as $\gamma$-ray bursts (GRBs). The explosive energy from a GRB is thought to be channelled into two narrow jets. Photon emission occurs in two stages: the prompt-emission phase and the afterglow phase. In the afterglow phase, low-energy photons are thought to be generated by a mechanism called synchrotron radiation. High-energy photons are thought to be mainly produced through a process dubbed synchrotron self-Compton (SSC), whereby the scattering of synchrotron photons off energetic electrons gives the photons a boost in energy ${ }^{5,7} . \mathbf{b}$, One key prediction of the SSC mechanism is that there should be two 'humps' in the spectral energy distribution of the afterglow spectrum: one corresponding to synchrotron photons and the other to SSC photons ${ }^{5,7}$. Results from the three papers firmly establish the existence of such an SSC component.

structure has been commonly observed for high-energy jets launched from supermassive black holes known as blazars ${ }^{8}$, and the same structure has been confidently expected for GRBs. Previous observations of high-energy afterglows of GRBs using the Large Area Telescope on board the Fermi satellite have not convincingly shown the existence of a second hump in the spectral energy distributions ${ }^{9}$. However, some tentative evidence has been collected from another bright burst ${ }^{10-12}$, GRB 130427A.

The multi-wavelength observations of GRB 190114C obtained by the MAGIC Collaboration and colleagues have firmly established, for the first time, the existence of the SSC component in a GRB afterglow ${ }^{3}$. This conclusion has been confirmed by independent modelling from other groups ${ }^{13-15}$. The double-hump feature is comparatively less clear in the spectral energy distribution obtained by Abdalla et al. for GRB 180720B. However, in the late afterglow phase, electron synchrotron radiation cannot produce photons of energies above $100 \mathrm{GeV}$ without the need to introduce exotic particle-acceleration mechanisms. As a result, the SSC mechanism is the preferred explanation for the observed spectral energy distribution ${ }^{4,13}$

Why did it take so long to detect a theoretically expected common spectral component? The observation of a GRB by an IACT requires that the burst is bright (to produce a sufficient number of high-energy photons) and nearby (to avoid absorption of the photons by the infrared background radiation in the Universe). Furthermore, the telescope needs to have the correct observational conditions. For instance, a particular GRB would not be detected by an IACT if the event occurred during the daytime, in poor weather or in an area of the sky that was not accessible by the telescope. Nevertheless, the breakthrough results reported in the current papers suggest that, with dedication and probably a bit of luck, a revolutionary discovery can be made.

Now that photons of energies above $100 \mathrm{GeV}$ have been detected from GRBs, it is expected that such detections will become routine in the future - especially with the full operations of the available IACTs and of observatories that use other detection techniques, such as the High-Altitude Water Cherenkov Observatory in Mexico. The field will also greatly benefit from the operations of facilities such as the future international Cherenkov Telescope Array and the Large High Altitude Air Shower Observatory in Daocheng, China. As history has repeatedly shown, the opening of a new spectral window in GRB research always reveals many treasures for researchers to mine. This spectral window at the highest energies will not be any different, and could be even more rewarding.

Bing Zhang is in the Department of Physics and Astronomy, University of Nevada, Las Vegas, Nevada 89154, USA. e-mail: zhang@physics.unlv.edu

\footnotetext{
1. Zhang, B. The Physics of Gamma-Ray Bursts (Cambridge Univ. Press, 2018)

MAGIC Collaboration. Nature 575, 455-458 (2019)

. MAGIC Collaboration et al. Nature 575, 459-463 (2019).

Abdalla, H. et al. Nature 575, 464-467 (2019).

Zhang, B. \& Mészáros, P. Astrophys. J. 559, 110-122 (2001).

6. Racusin, J. L. et al. Astrophys. J. 738, 138 (2011).

7. Sari, R. \& Esin, A. A. Astrophys. J. 548, 787-799 (2001).

8. Ghisellini, G., Righi, C. Costamante, L. \& Tavecchio, F. Mon. Not. R. Astron. Soc. 469, 255-266 (2017).

9. Kumar, P. \& Barniol Duran, R. Mon. Not. R. Astron. Soc. 400, L75-L79 (2009).

10. Ackermann, M. et al. Science 343, 42-47 (2014).

11. Liu, R.-Y., Wang, X.-Y. \& Wu, X.-F. Astrophys. J. 773, L2O (2013).

12. Fan, Y.-Z. et al. Astrophys. J. 776, 95 (2013)

13. Wang, X.-Y., Liu, R.-Y., Zhang, H.-M., Xi, S.-Q. \& Zhang, B. Astrophys. J. 884, 117 (2019).

14. Derishev, E. \& Piran, T. Astrophys. J. 880, L27 (2019).

15. Fraija, N. et al. Astrophys. J. 883, 162 (2019).
} 